\title{
Comparison of Discrete Cosine Transforms (DCT), Discrete Fourier Transforms (DFT), and Discrete Wavelet Transforms (DWT) in Digital Image Watermarking
}

\author{
Rosa A Asmara \\ Information Technology Department \\ State Polytechnics of Malang \\ Malang, Indonesia
}

\author{
Reza Agustina \\ Information Technology Department \\ State Polytechnics of Malang \\ Malang, Indonesia
}

\author{
Hidayatulloh \\ Information Technology Department \\ State Polytechnics of Malang \\ Malang, Indonesia
}

\begin{abstract}
Digital Image Watermarking is used recently to secure the image by embedding another digital image. It is typically used to identify ownership of the copyright of the signal. Frequency domain transformation methods used widely in Digital Image Compression and Digital Image Watermarking. They reduce the weakness of classics digital image watermarking such as Least Significant Bit (LSB) methods which is more noisetolerant. Popular transformation method used are Two Dimensional Discrete Cosine Transform (2D DCT), Two Dimensional Discrete Fourier Transforms (2D DFT), and Two Dimensional Discrete Wavelet Transform (2D DWT). This paper will show the comparison result of those three transformation method. The experiments are comparison analysis of image watermark quality using Peak Signal to Noise Ratio (PSNR), color converting, image resizing, image optical scanning and the noise-tolerant of the image watermarked by giving Gaussian noise.
\end{abstract}

Keywords-Digital Image Watermarking; $2 D$ Discrete Cosine Transform (2D DCT); 2D Discrete Fourier Transform (2D DFT); 2D Discrete Wavelet Transform (2D DWT); Least Significant Bit method (LSB); Digital Signal Processing

\section{INTRODUCTION}

Digital Image Watermarking is used recently to secure the image by embedding another digital image. Komatsu and Tominaga are the first person using the term Digital Watermarking [1]. It is typically used to identify ownership of the copyright of the signal. The information are embedded in image is called a "digital image watermark". The information where the watermark is to be embedded is called a "host image" [2,3]. Traditional method for Digital Image Watermarking used Least Significant Bit (LSB). Many researcher proposed the LSB method with some improvement and analysis to create better digital image watermark results $[4,5,6]$. The method for LSB will be explained in detail in Section 2.

LSB method for digital image watermarking has a weakness which cannot handle simple noise. Image watermarked also will loss the watermark information if some image processing is implemented such as Image Resizing and Image Cropping. Some Frequency Domain Transformations method is implemented to handle such weakness in traditional method. It is also proved that even the image is printed and scanned to return the format in digital, the watermark image can also be extracted smoothly.

Discrete Cosine Transform (DCT) are popular among science and engineering application, from image watermarking, steganography, and lossy compression for audio and image. Cosine function is used rather than sine function due to the critical for compression, fewer cosine functions are needed to approximate the typical signal. Cosine functions also express a particular choice of boundary condition in differential equations. DCT is similar to the Discrete Fourier Transform (DFT), but using only real numbers. DCT are equivalent of DFT of roughly twice the length, operating on real data with even symmetry and in some variants the input or output data are shifted by half a sample.

The remainder of this paper is organized as follows: Section 2 explained the 2D DCT, 2D DFT, 2D DWT and PSNR for analyzing the watermarked image. Section 3 describes our experiment and a technique for image watermarking in frequency domain information. Section 4 presents the experiments results. Conclusion and future work are given in the final section.

\section{PROPOSED METHOD}

\section{A. Digital Image Watermarking}

Digital image watermarking is one of the steganography branches, which is a technic to hide information in a digital media. The purpose is to protect important information [8]. The information inserted in digital image watermarking can be in text, image, or audio format file. There are some criteria to create good image watermarking:

- Imperceptibility: Good Watermark is invisible by human eye. One cannot distinguish well between original and watermarked image.

- Robustness: Good Watermark has to be resisted with file manipulation such as file compression, image noising, color converting, and image resizing.

- Security: Watermarked file can only be detected by the file owner or authorized ones. 
- Recovery: Watermarked file must be converted back to original file. Main purpose of watermarking is for copyrighting file owner, which can be used for file authorization.

\section{B. Least Significant Bit Method}

Least significant bit (LSB) is very simple and less computation cost compare to the other method of transformations. Figure 1 shows the insertion method in LSB. For every bit starting from MSB of watermark image pixel is inserted to the LSB of some image source pixel. The results will be the addition of 1 if the watermark bit is 1 and same as previous value if the watermark bit is 0 . Human eyes will be difficult to distinguishing the image source before and after watermarking. However, this method faces some image processing implementations such as Image Resizing and Image Cropping due to the pixel value modification. The PSNR of the LSB 1 bit substitution is 55.8784 [4].

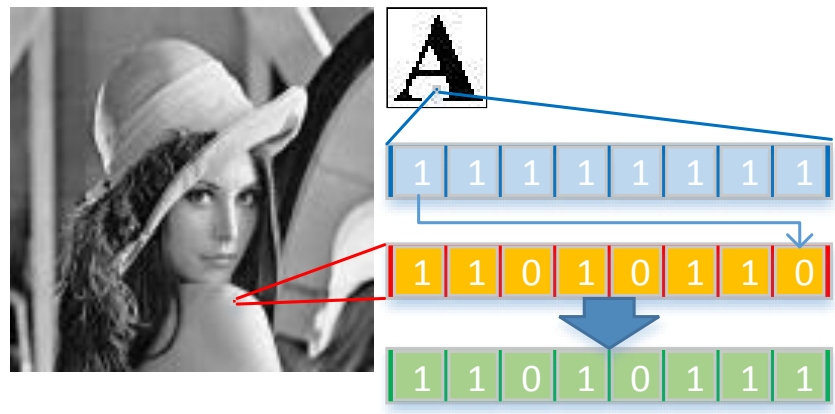

Fig. 1. LSB method of Digital Image Watermarking

\section{2D Discrete Cosine Transform (2D DCT)}

DCT in image processing is first introduced by Ahmed, Natarajan and Rao [7]. DCT is similar to DFT, but using only real numbers. DCT turn over the image edge to make the image transformed into other form of even function. This is one of linear transformations in digital signal processing. 2D DCT is defined as:

$$
\begin{array}{r}
F(j k)= \\
a(j) a(k) \sum_{m=0}^{N-1} \sum_{n=0}^{N-1} f(m n) \cos \left[\frac{(2 m+1) j \pi}{2 N}\right] \cos \left[\frac{(2 n+1) k \pi}{2 N}\right] \quad[1]
\end{array}
$$

The corresponding inverse discrete cosine transformation (2D-IDCT) is defined as:

$$
\begin{array}{r}
f(m n)= \\
\sum_{m=0}^{N-1} \sum_{n=0}^{N-1} a(j) a(k) F(j k) \cos \left[\frac{(2 m+1) j \pi}{2 N}\right] \cos \left[\frac{(2 n+1) k \pi}{2 N}\right]
\end{array}
$$

The 2D-DCT can not only focusing on transform the main information of original image into the smallest low-frequency component, but also it can cause the image blocking effect being the smallest, which can realize the good compromise between the information centralizing and the computing complication, thus it obtains the wide spreading application.

\section{2D Discrete Fourier Transform (2D DFT)}

Consider one N1 x N2 image, $\mathrm{f}(\mathrm{n} 1, \mathrm{n} 2)$, where we assume that the index range are $n_{1}=-M_{1}, \ldots, M_{1}$ and $n_{2}=-M_{2}, \ldots, M_{2}$, for mathematical simplicity, and hence $\mathrm{N}_{1}=2 \mathrm{M}_{1}+1$ and $\mathrm{N}_{2}=$
$2 \mathrm{M}_{1}+1$. Let $\mathrm{F}\left(\mathrm{k}_{1}, \mathrm{k}_{2}\right)$ denote the $2 \mathrm{D}$ discrete Fourier Transform (2D DFT) of the image. $\mathrm{F}\left(\mathrm{k}_{1}, \mathrm{k}_{2}\right)$ are given by

$$
\begin{array}{r}
F\left(k_{1}, k_{2}\right)= \\
\sum_{n_{1} n_{2}} f\left(n_{1}, n_{2}\right) W_{N_{1}}^{k_{1} n_{1}} W_{N_{2}}^{k_{2} n_{2}}=A_{F}\left(k_{1}, k_{2}\right) e^{j \theta_{F}\left(k_{1}, k_{2}\right)}
\end{array}
$$

Where $\mathrm{k}_{1}=-\mathrm{M}_{1}, \ldots, \mathrm{M}_{1}, \mathrm{k}_{2}=-\mathrm{M}_{2}, \ldots, \mathrm{M}_{2}, W_{N_{1}}=e^{-j \frac{2 \pi}{N_{1}}}$, $W_{N_{2}}=e^{-j \frac{2 \pi}{N_{2}}}$, and the operator $\sum_{n_{1} n_{2}}$ denotes $\sum_{n_{1}=-M_{1}}^{M_{1}} \sum_{n_{2}=-M_{2}}^{M_{2}}, A_{F}\left(k_{1}, k_{2}\right)$ is an amplitude component, and $e^{j \theta_{F}\left(k_{1}, k_{2}\right)}$ is a phase component.

The 2D Inverse Discrete Fourier (2D IDFT) of $F\left(k_{1}, k_{2}\right)$ is given by

$f\left(k_{1}, k_{2}\right)=\frac{1}{N_{1} N_{2}} \sum_{k_{1} k_{2}} F\left(k_{1}, k_{2}\right) W_{N_{1}}^{-k_{1} n_{1}} W_{N_{2}}^{-k_{2} n_{2}}$

Where $\sum_{k_{1} k_{2}}$ denotes $\sum_{k_{1}=-M_{1}}^{M_{1}} \sum_{k_{2}=-M_{2}}^{M_{2}}$.

\section{E. 2D Discrete Wavelet Transform (2D DWT)}

Discrete wavelet transform (DWT) represents an image as a subset of wavelet functions using different locations and scales. It makes some decomposition images. Any decomposition of an image into wavelet involves a pair of waveforms: the high frequencies corresponding to the detailed parts of an image and the low frequencies corresponding to the smooth parts of an image. DWT for an image as a 2-D signal can be derived from a 1-D DWT. According to the characteristic of the DW decomposition, an image can be decomposed to four sub-band images through a 1-level 2-D DWT, as shown in Fig. 2. These four sub-band images in Fig. 4 can be mapped to four sub-band elements representing LL (Approximation), HL (Vertical), LH (Horizontal), and $\mathrm{HH}$ (Diagonal) respectively.

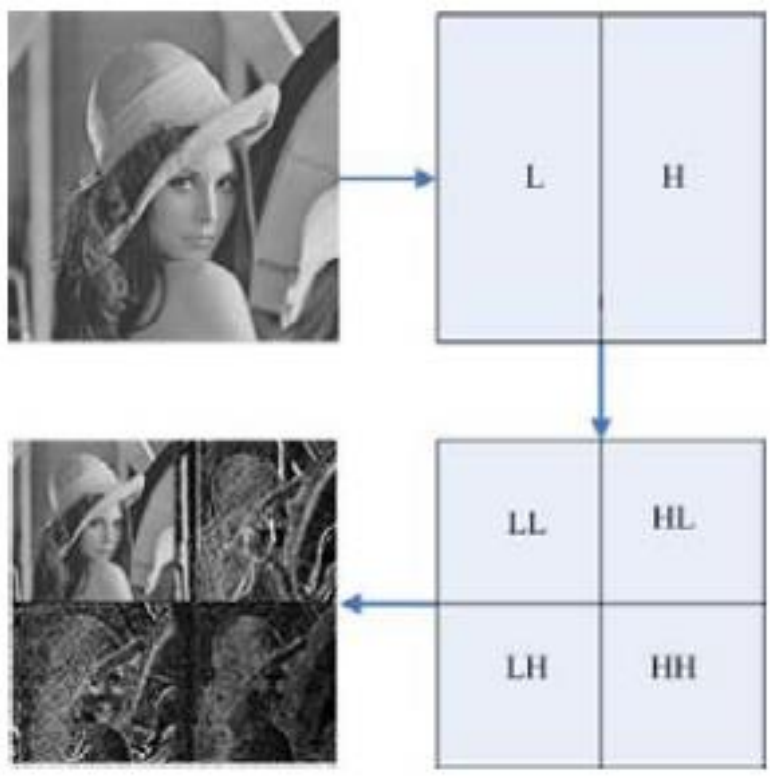

Fig. 2. 1-Level Decomposition 2D DWT

The discrete Wavelet Transform will decompose a given signal into other signal known as the approximation and detail coefficients. A given function $\mathrm{f}(\mathrm{t})$ can be expressed through the following representation: 
$\sum_{\mathrm{j}=1}^{\mathrm{L}} \sum_{\mathrm{K}=-\infty}^{\infty} \mathrm{d}(\mathrm{j}, \mathrm{K}) \varphi\left(2^{-\mathrm{j}} \mathrm{t}-\mathrm{K}\right)+\sum_{\mathrm{K}=-\infty}^{\infty} \mathrm{a}(\mathrm{L}, \mathrm{K}) \theta\left(2^{-\mathrm{L}} \mathrm{t}-\mathrm{K}\right)$

Where: $\varphi(t)$ is the mother wavelet and $\theta(\mathrm{t})$ is the scaling function. $a(\mathrm{~L}, \mathrm{~K})$ is called the approximation coefficient at scale $\mathrm{L}$ and $\mathrm{d}(\mathrm{j}, \mathrm{K})$ is called the detail coefficients at scale $\mathrm{j}$. The approximation and detail coefficients can be expressed as:

$\mathrm{a}(\mathrm{L}, \mathrm{K})=\frac{1}{\sqrt{2^{\mathrm{L}}}} \int_{-\infty}^{\infty} \mathrm{f}(\mathrm{t}) \theta\left(2^{-\mathrm{L}} \mathrm{t}-\mathrm{K}\right) \mathrm{dt}$

$\mathrm{d}(\mathrm{j}, \mathrm{K})=\frac{1}{\sqrt{2^{\mathrm{j}}}} \int_{-\infty}^{\infty} \mathrm{f}(\mathrm{t}) \varphi\left(2^{-\mathrm{j}} \mathrm{t}-\mathrm{K}\right) \mathrm{dt}$

Based on the choice of the mother wavelet $\varphi(t)$ and scaling function $\theta(\mathrm{t})$, different families of wavelets can be constructed.

\section{F. Mean Square Error (MSE) and Peak Signal to Noise Ratio (PSNR)}

Peak signal-to-noise ratio (PSNR) is a ratio between maximum possible power of a signal and the power of corrupting noise that affects the fidelity of its representation. PSNR is usually expressed in terms of the logarithmic decibel scale. The signal is an original data, and the noise is the error from watermark system.

PSNR usually defined via the mean squared error (MSE). Given a noise-free $\mathrm{m} \times \mathrm{n}$ image I and noisy approximation K, MSE is defined as:

$$
M S E=\frac{1}{M N} \sum_{i=0}^{M-1} \sum_{j=0}^{N-1}(I(i, j)-K(i, j))^{2}
$$

The PSNR (in $\mathrm{dB}$ ) is defined as:

$$
\begin{aligned}
\text { PSNR } & =10 \log _{10}\left(\frac{M A X_{I}^{2}}{M S E}\right) \\
& =20 \log _{10}\left(\frac{M A X_{I}}{\sqrt{M S E}}\right) \\
& =20 \log _{10}\left(M A X_{I}\right)-10 \log _{10}(M S E)
\end{aligned}
$$

Where, $\mathrm{MAX}_{\mathrm{I}}$ is the maximum possible pixel value of the image. When the pixels are represented using 8 bits per sample, this is 255 .

\section{EXPERIMENTS}

Figure below shows the general watermark image steps in frequency domain transforms.

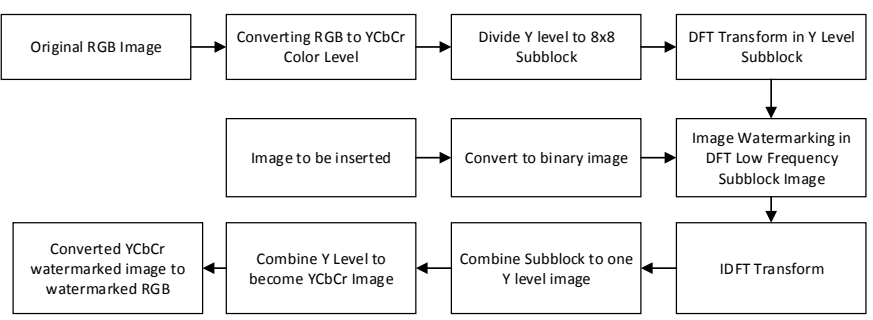

Fig. 3. Watermark image steps in frequency domain transform

The usage of DCT, DFT, and DWT in image watermarking start by dividing image to $8 \times 8$ pixel sub-block. These subblock will consist of 64 coefficient (1 DC-zero frequency coefficient and $63 \mathrm{AC}$ coefficient in low frequency, middle frequency, and high frequency. Figure 4 shows the DCT frequency division.

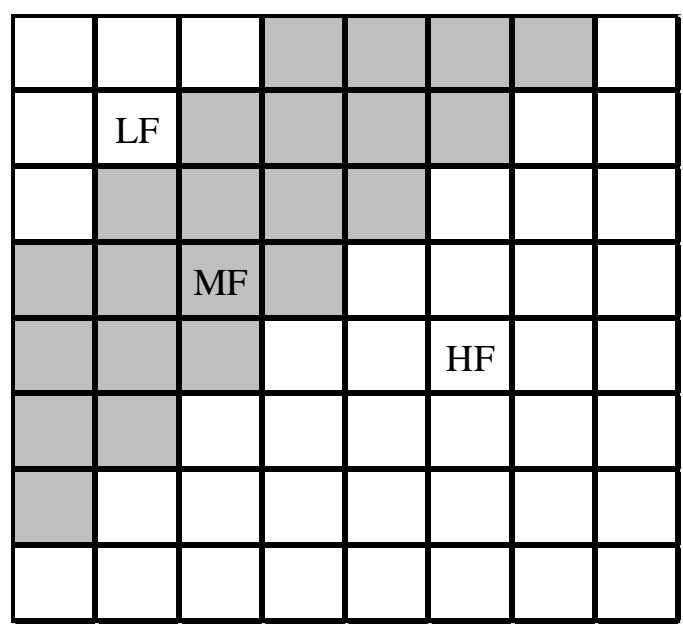

Fig. 4. DCT Frequency components

LF represents the low frequency, MF represents middle frequency, and HF represents sub-block highest frequency. In DCT transform, LF can be found in every edge and corner of image sub-block. In DCT and DFT, watermark bit image will be put in LF. In DWT, watermark bit image will be put in LH and HL. Figure 5 shows the diagram block for DCT watermarking process. This diagram block also represents for DFT and DWT. 


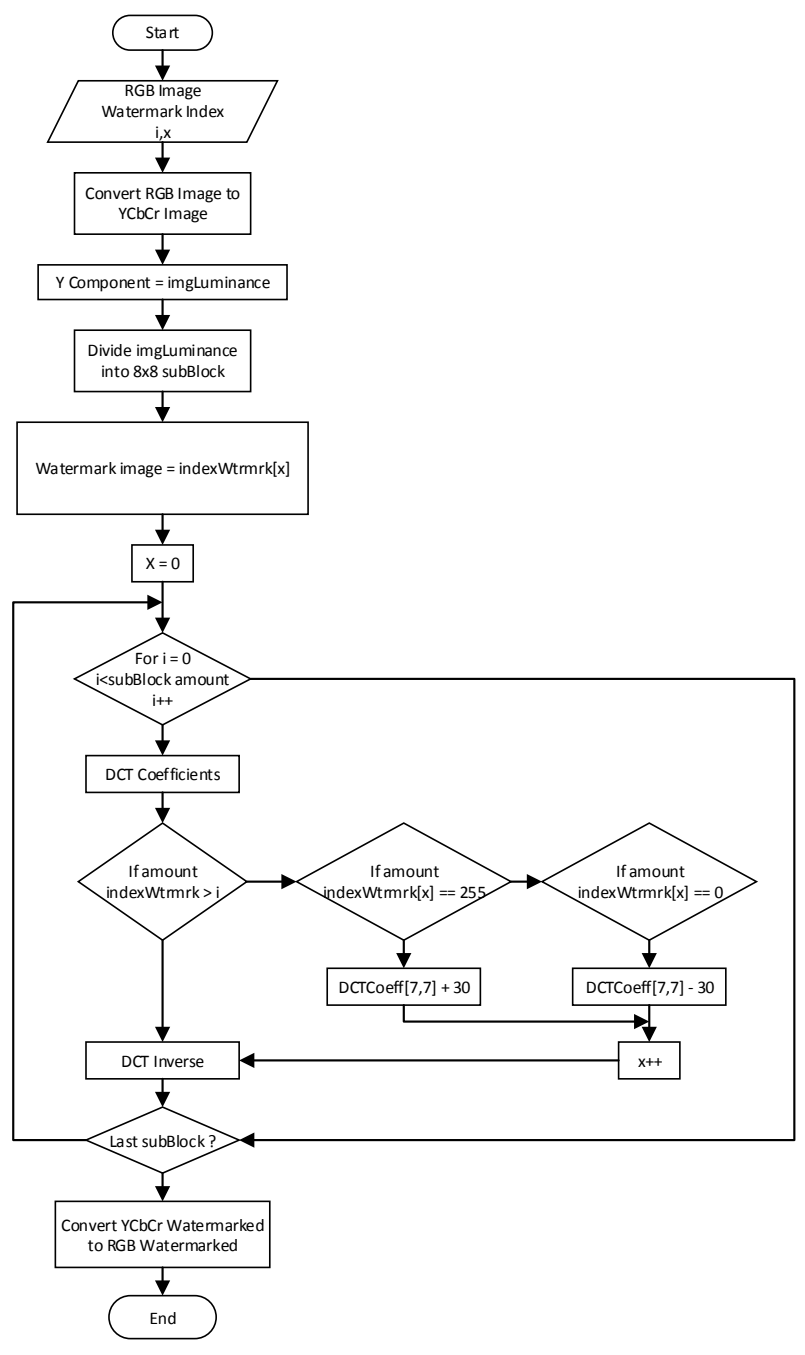

Fig. 5. Diagram blocks of DCT image watermarking

- User input host image, RGB image in which watermark image will be inserted. Watermark image will use binary image provided.

- RGB image will be converted to YCbCr color space, Y color space (luminance) component is the only component where the watermark image will be inserted, and thus the Y-component transformed using DCT, DFT, and DWT. Y-component is used because human perception more sensitive to the light intensity and the result for watermarked image will imperceptible. Chrominance blue (Cb) and chrominance red $(\mathrm{Cr})$ component will not transform and will be used only for Inverse Transform.

- Y-component is divided into 8x8 sub-blocks.

- Each 8x8 sub-blocks then transforms using DCT, DFT, and DWT. Image watermark is a binary image with 0 and 1 pixel value. If the pixel value is 1 in the watermark image, then the sub-block host image in index [7,7] will be add with 30 , otherwise will be subtract with 30 . This process is then repeated until the last watermark image pixel. The Y-component then detransformed using inverse-DCT, inverse-DFT, and inverse-DWT to get Y-component watermarked image.

- Y-component is combined again with $\mathrm{Cb}$-component and $\mathrm{Cr}$-component to get $\mathrm{YCbCr}$ watermarked image.

- Last proses is YCbCr watermarked converted again to RGB Space to get RGB watermarked.

\section{RESULTS}

We use $32 \times 32$ binary image resolutions as an image watermark. This image will embed on image with 65536 total pixel amount, since 1 pixel of binary image will embed on $8 \times 8$ image sub-block. Figure 5(a) is used as an image watermark and figure 5(b) as an original image.

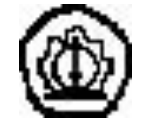

(a)

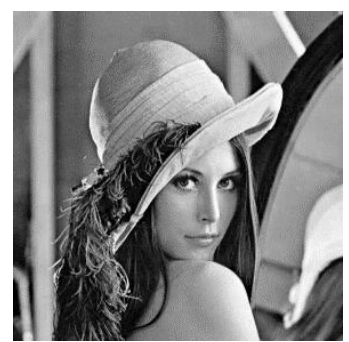

(b)
Fig. 6. (a) Image Watermark, (b) Original Image

Table 1 shows the results of embedding image watermark to the LF, MF, and HF of original image.

TABLE I. PSNR RESULTS OF IMAGE WATERMARK IN DCT, DFT, AND DWT

\begin{tabular}{|l|l|l|l|}
\hline $\begin{array}{l}\text { Freq.: Embed } \\
\text { Pixel Coordinate }\end{array}$ & $\begin{array}{l}\text { DCT } \\
\text { PSNR } \\
(\mathrm{dB})\end{array}$ & $\begin{array}{l}\text { DFT } \\
\text { PSNR } \\
(\mathrm{dB})\end{array}$ & $\begin{array}{l}\text { DWT PSNR } \\
(\mathrm{dB})\end{array}$ \\
\hline LF: [w0, h1] & 40,24 & 32,2 & 33,78 \\
\hline MF: [w3, h3] & 39,96 & 35,18 & $\begin{array}{l}33,87(\mathrm{HL}) \\
33,87(\mathrm{LH})\end{array}$ \\
\hline HF: $[\mathrm{w} 7, \mathrm{~h} 7]$ & 40,82 & 32,18 & 33,87 \\
\hline
\end{tabular}

TABLE II. PSNR RESULTS OF GAUSSIAN NOISE ATTACK IN WATERMARK DCT, DFT, AND DWT

\begin{tabular}{|l|l|l|l|}
\hline \multicolumn{4}{|c|}{$20 \%$ Gaussian Noise to the watermarked image } \\
\hline Frequency & $\begin{array}{l}\text { PSNR } \\
\text { DCT }\end{array}$ & $\begin{array}{l}\text { PSNR } \\
\text { DFT }\end{array}$ & PSNR DWT \\
\hline LF & 19,36 & 19,04 & 18,90 \\
\hline MF & 19,26 & 19,00 & $\begin{array}{l}19,01(\mathrm{HL}) \\
18,95(\mathrm{LH})\end{array}$ \\
\hline HF & 19,29 & 19,11 & 19,15 \\
\hline
\end{tabular}

TABLE III. PSNR RESULTS OF COMPRESSION ATTACK IN WATERMARK DCT, DFT, AND DWT

\begin{tabular}{|c|c|c|c|}
\hline \multicolumn{4}{|c|}{ Compression test using RIOT Application } \\
\hline Level & $\begin{array}{c}\text { Extraction } \\
\text { DCT }\end{array}$ & Extraction DFT & $\begin{array}{c}\text { Extraction } \\
\text { DWT }\end{array}$ \\
\hline $25 \%$ & (1) & (1) \\
\hline $50 \%$ & & \\
\hline
\end{tabular}


TABLE IV. PSNR RESULTS OF IMAGE CONTRAST MANIPULATION IN WATERMARK DCT, DFT, AND DWT

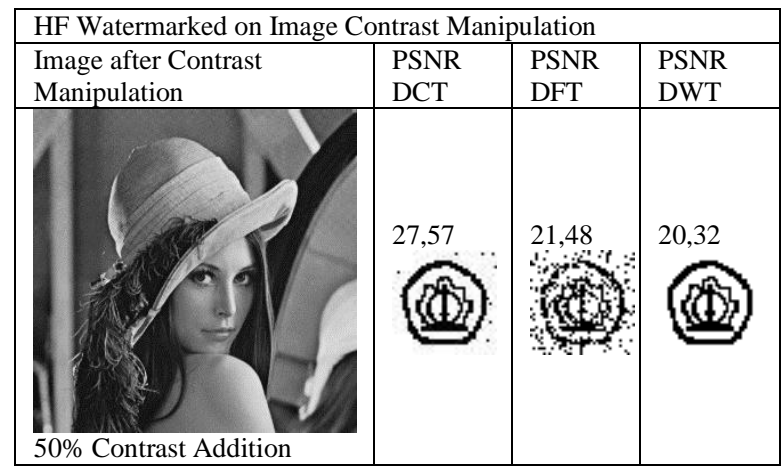

\section{CONCLUSION}

Frequency domain transformation methods are used widely in Digital Image Compression and Digital Image Watermarking. It reduces the weakness of classics digital image watermarking such as Least Significant Bit (LSB) methods which is more noise-tolerant. Popular transformation method used are Two Dimensional Discrete Cosine Transform (2D DCT), Two Dimensional Discrete Fourier Transforms (2D DFT), and Two Dimensional Discrete Wavelet Transform (2D DWT). This paper proposed the comparison between those three transformation methods. The experiments are image watermark quality analysis using Mean Square Error (MSE), Peak Signal to Noise Ratio (PSNR), and the noise-tolerant of the image watermarked by giving Gaussian noise in it. The experiments shows result of:

- DCT Transformation embed on High Frequency is the best for image watermarking. It has $40.82 \mathrm{~dB}$ PSNR values, as shown in table 1 .

- Adding 20\% Gaussian noise to the watermarked image, the best Transformation for Gaussian Attack is DCT watermark in Low Frequency. It has $19.36 \mathrm{~dB}$ PSNR value, as show in table 2 .

- Compressing image using RIOT application for all three transformation, DWT shows the best results as shown in table 3.

Using image contrast manipulation, DCT shows the best results as shown in table 4.

\section{REFERENCES}

[1] Bender, W., Gruhl, D., Morimoto, N. and Lu, A(1996).: Techniques for data hiding. IBM Systems Journal, vol. 35, nos. 3\&4.

[2] Saraju Prasad Mohanty(,January 1999 )"Watermarking of Digital Images", Submitted at Indian Institute of Science Bangalore, pp. 1.3 1.6,.

[3] Katzenbeisser, S. and Petitcolas, F(1999).: Information hiding techniques for steganography and digital watermarking. Artech House Books.

[4] Puneet Kr Sharma and Rajni(2012): ANALYSIS OF IMAGE WATERMARKING USING LEAST SIGNIFICANT BIT ALGORITHM, International Journal of Information Sciences and Techniques (IJIST) Vol.2, No.4, July 2012.

[5] Abdullah Bamatraf, Rosziati Ibrahim and Mohd. Najib Mohd. Salleh(2011): A New Digital Watermarking Algorithm Using Combination of Least Significant Bit (LSB) and Inverse Bit, Journal of Computing Vol.3, Issue 4, April 2011, ISSN 2151-9617.

[6] Deepshikha Chopra, Preeti Gupta, Gaur Sanjay B.C., Anil Gupta (2012): Lsb Based Digital Image Watermarking For Gray Scale Image, IOSR Journal of Computer Engineering (IOSRJCE) Vol.6, Issue 1, pp 36-41, Sep-Oct 2011, ISSN 2278-0661.

[7] Ahmed, Natarajan, and Rao (1974) : On Image Processing and a Discrete Cosine Transform, IEEE Trans. On Computer C-23(1): 90-93

\section{AUTHOR PROFILE}

Rosa A. Asmara: Lecturer of Information Technology Department in State Polytechnics of Malang, Indonesia. Research interest are Image Processing and Computer Vision, Pattern Recognition, and Artificial Intelligence.

Reza Agustina, Hidayatulloh: Undergraduate student in Information Technology Department, State Polytechnics of Malang, Indonesia. 